Sir,

\section{Reply to Dr Hoffer}

Dr Hoffer's comments are very important. The queries are discussed individually:

The question of limitation on the ACD: In my paper I discussed results taking the input data $\mathrm{AL}=23.45$, $K=45, \mathrm{ACD}$ constant $=6.15$ (personalised or manufacturer's, whatever), and vertex $=12$, giving a predicted anterior chamber depth $=6.14334$. In no case was there any need to go to any extremes of either AL or $\mathrm{ACD}$. So the question of limiting the value of ACD does not arise. Even without going into any detail, it is possible to test whether these limitations on ACD are being executed in my program with the given input values, merely by placing flags in the 'if statements' in the HofferQ function in my code, and analysing the output.

The missing minus sign: This printing error in the original paper was corrected in a subsequent published errata. This factor has been taken into account as shown in the function for Hoffer $Q$ in my paper.

The retinal thickness factor: As my computer code shows, the retinal thickness factor $R_{-}$th was considered for Holladay I and SRK-T (in different ways, of course) but not for Hoffer Q.

The question of checking with the formula author or the manufacturer: On recommendation of the Eye editorial board, the article was sent before publication to both the formula author and Carl Zeiss Meditech, and their valuable comments were taken into consideration in this article. So the comment about not validating the formula with the author(s) is premature, injudicious and deplorable.

It is best to reiterate here that IOLMaster's accuracy for biometry and HofferQ formula's predictive power have been established well. The point in question in the article, however, is whether the IOLMaster is as accurate in implementing the IOL formulas or if there is scope of any error. This paper is an attempt in that direction. To label this attempt as 'blatantly detrimental' is a gross injustice to any scientific criticism. Unfortunately, while the aforesaid main issue remains unsolved, the focus of discussion has digressed elsewhere.

It is painful to hear that one should use only an 'approved and licensed' version of a formula, and not the one that is published in the public domain. It makes one wonder where would science be today if Einstein's $E=m c^{2}$ or Newton's laws of gravitation or Kepler's laws of planetary motion were all copyrighted.
S Basu

William Harvey Hospital,

Kennington Road,

Willesborough,

Ashford, Kent TN24 OLZ, UK

Correspondence: S Basu,

Tel: +44 1233 633331, ext. 88534;

Fax: + 441233616770 .

E-mail: sbasu2003@doctors.net.uk

Eye (2007) 21, 430. doi:10.1038/sj.eye.6702561;

published online 29 September 2006

Sir,

Reply to Basu S et al

With great interest I read the paper on IOLMaster vs theoretical calculations in Eye (2006) 20: 90-97.

My experience, however, with the HofferQ implementation and the IOLMaster is different and I wonder what the reasons might be.

Looking at the code at the bottom of page 95, in the Function HofferQ, there is the statement $A C D=A$ toACD(ELPtoA(ELPconst)). The variable $A C D$ is later used to calculate the predicted ACD in $\mathrm{ACD}=\mathrm{ACD}+0.3^{*} \ldots$

The 'ACD' on the right side of this equation/definition actually should be the HofferQ IOL constant ' $\mathrm{pACD}$ ', that is, in the expression $\mathrm{ACD}=\mathrm{Ato} A C D(E L P t o A(E L P c o n s t))$ 'ACD' should play the role of ' $\mathrm{pACD}$ ' in the original HofferQ publication. Why convert ELPconst $\rightarrow \mathrm{A} \rightarrow \mathrm{ACD}$ ?

Trying to reproduce the example 1 (emmetropic eye $A=23.5, K=43.5)$ ) in Hoffer's original paper of 1993, the predicted $\mathrm{ACD}$ for an IOL constant $\mathrm{PACD}=4.50$ should be 4.40887 .

Assuming that the variable ELPconst stands for pACD then I have a problem understanding the conversion ELPconst $\rightarrow \mathrm{A} \rightarrow \mathrm{ACD}$. $\mathrm{ACD}=$ AtoACD(ELPtoA (ELPconst)) would turn out to be 4.46591 instead of 4.50 and the predicted Hoffer ACD would be 4.37479 instead of 4.40887. Could this explain the problems that were observed? The function HofferQ needs the entry of whatever stands for the paper's 'pACD' in the nomenclature. Which variable in the code plays this role?

W Haigis

Department of Ophthalmology, University of Wuerzburg, Sanderring, Wuerzburg D-97070, Germany 
Correspondence: W Haigis,

Tel: + 49931201 20640;

Fax: + 4993120120454

E-mail:w.haigis@augenklinik.uni-wuerzburg.de

Eye (2007) 21, 430-431. doi:10.1038/sj.eye.6702562;

published online 29 September 2006

Sir,

Fabry disease manifesting as chronic uveitis - treated with enzyme replacement therapy

Fabry disease, a metabolic disease, is caused by $\alpha$-galactosidase A deficiency. ${ }^{1}$ Its ophthalmic manifestations include vortex keratopathy, cataract, and retinal vessel tortuosity. ${ }^{2}$ We report a case of Fabry disease presenting with chronic uveitis, cystoid macular oedema, and marked visual impairment. It responded only temporarily to periocular steroid injections and was finally stabilized under enzyme replacement therapy (ERT). To our knowledge, these unusual manifestations and the treatment have never been reported.

\section{Case report}

A 22-year-old man visited us in May 2002 and complained of progressive decreased vision (OU) for 4 years, along with acroparesthesia, hypohidrosis, and heat intolerance. Best-corrected visual acuity (BCVA) was 20/400 (OD) and 20/200 (OS). Ophthalmological exams showed bilateral vortex keratopathy, anterior chamber reaction, anterior subcapsular cataract, vitreous haze, periarterial infiltrate, cystoid macular oedema (Figure 1a and b), and diffuse vascular leakage (Figure 1c and d). Optical coherence tomography (OCT) showed severe macular oedema (Figure 1e and f). Fabry disease was confirmed by enzymatic analysis. Investigations looking for other causes of uveitis were all negative.

Because the clinical condition progressed, a therapeutic trial with posterior subtenon steroid injection (triamcinolone acetonide $20 \mathrm{mg}$, OU) was performed. Two weeks later, BCVA improved to 20/70 (OD) and 20/100 (OS). The vitreous haze and macular oedema partially resolved (Figure $1 \mathrm{~g}$ and $\mathrm{h}$ ). However, the inflammation waxed and waned despite subsequent periocular steroid injections. BCVA decreased to counting fingers at $40 \mathrm{~cm}(\mathrm{OU})$ owing to macular oedema, cataract, and severe vitreous haze. He received ERT (agalsidase alfa, $10.5 \mathrm{mg}$ every 2 weeks from September 2002 to November 2003; agalsidase beta, 70 mg every 2 weeks since December 2003) and cataract extraction (OD, November 2002; OS, May 2003). After 24 weeks of ERT, acroparesthesia, hypohidrosis, and heat intolerance relieved and the uveitis-like picture gradually subsided. Subsequently, he underwent pars plana vitrectomy (OD) for persistent severe vitreous opacity. Postoperatively, the macula showed mottling change without oedema (Figure 2a). Although no inflammatory signs such as vitreous cells were noted in the left eye, severe vitreous opacity still obscured the fundus and probably affected the vision (Figure $2 \mathrm{~b}$ ). The latest BCVA was 20/400 (OD) and 20/800 (OS).

\section{Comment}

The most common retinal manifestation of Fabry disease is large vessel tortuosity. ${ }^{2}$ In contrast, this case presented with uveitis, vitreous haze, vascular leakage, cystoid
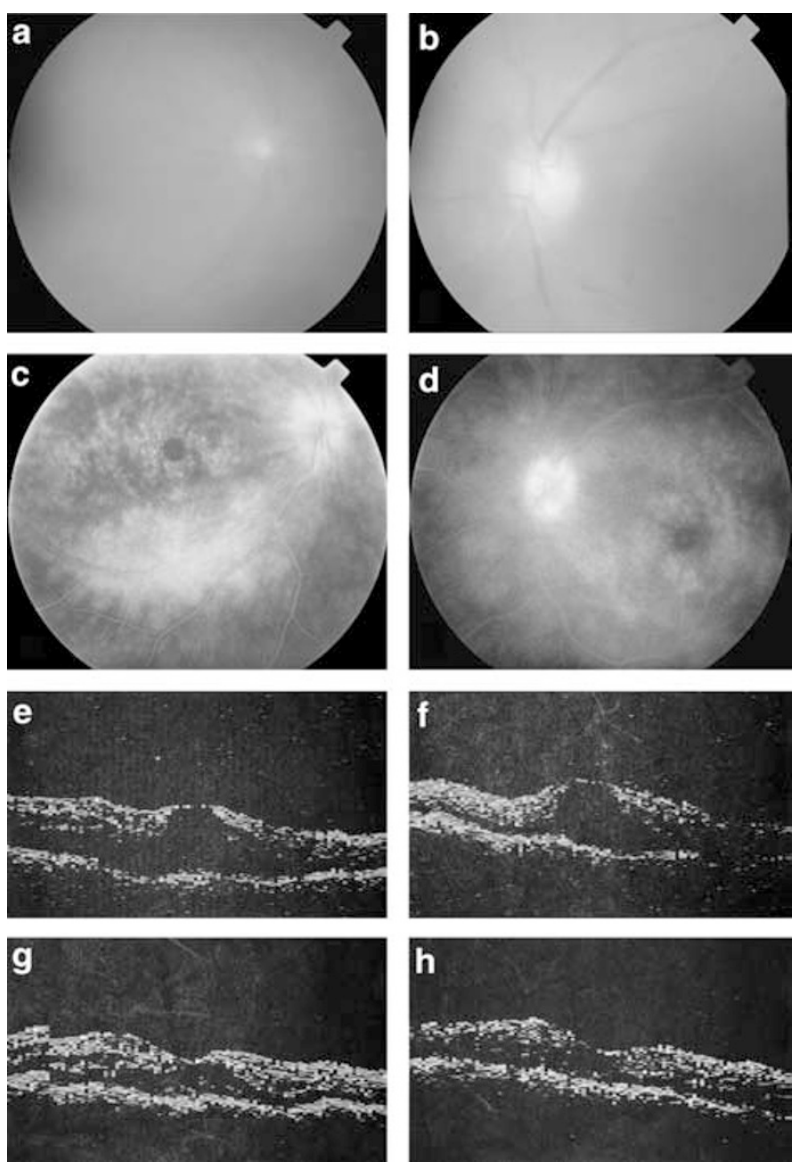

Figure $1 \quad(a, b)$ Fundus photography showed the vitreous haze obscuring the retina. (c, d) Fluorescein angiography showed vascular leakage, leakage from the optic disc, and cystoid macular oedema. (e, f) OCT before posterior subtenon steroid injection showed thickening of macula (e, OD; f, OS). (g, h) OCT showed significant decrease in macular thickness after posterior subtenon steroid injection ( $\mathrm{g}$, OD; h, OS). 